\title{
OBSERVER BASED FEEDFORWARD AIR-FUEL CONTROL OF TURBOCHARGED SI-ENGINES
}

\author{
Per Andersson and Lars Eriksson
}

\author{
Vehicular Systems, ISY \\ Linköping University, SE-581 83 Linköping, SWEDEN \\ Email: \{peran,larer\}@isy.liu.se
}

\begin{abstract}
Air-fuel control on turbocharged (TC) SI-engines require precise prediction of the cylinder air-charge (CAC). Using an observer it is possible to both estimate the necessary system states and to provide a framework to design the necessary CAC feedforward controller. Here a mean value engine model of a TC SI-engine is used to develop an observer. The output of the observer is fed as an initial condition to a predictor which is used for feedforward of the CAC for air-fuel control. The resulting controller is experimentally validated on a SAAB $2.0 \mathrm{dm}^{3} \mathrm{TC}$ engine using tip-in and tip-out transients. The results show that the excursions in $\lambda$ are less than 5\%. Copyright (C)2005 IFAC
\end{abstract}

Keywords: automotive control, automotive emissions, engine management, engine modeling, estimators, extended kalman filters, model based control

\section{INTRODUCTION}

In spark ignited (SI) engines it is essential to provide accurate air-fuel control to successfully reduce emissions using a three way catalyst (Heywood, 1988; Bauer et al., 1996; Kiencke and Nielsen, 2000; Mondt, 2000). In SI-engines the air-fuel ratio is controlled by the injected fuel mass. The injected fuel mass is determined from estimates of the cylinder air charge (CAC). As the injection is normally finished when the induction stroke starts, it is necessary to predict the $\mathrm{CAC}$ at the end of the induction stroke to avoid large errors in the air-fuel ratio during transients, see Figure 1.

The signals involved in the CAC calculation are subjected to noise, see Figure 2, and therefore needs filtering. Thus both prediction capabilities and filtering are necessary for accurate air-fuel control during transients.

Observers provide both filtering and prediction capabilities and these have successfully been used for CAC estimation on naturally aspirated engines (Powell et al., 1998; Choi and Hedrick, 1998). Here turbocharged (TC) engines are considered which have a more com-
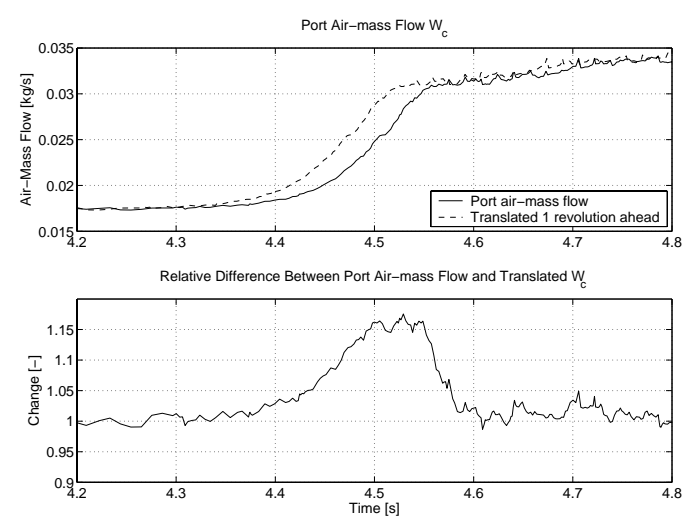

Fig. 1. A measured step in throttle at constant engine speed 1800 RPM where the port air mass flow (solid) is compared to a translated, corresponding to perfect prediction one revolution ahead, port air-mass flow. The difference between the curves is the resulting error if no prediction is used when the fuel mass is determined and in this case it would result in a $15 \%$ error in $\lambda$.

plex air-system where the turbocharger connects the intake side to the exhaust side through the turbine shaft and the CAC has a dependency on the exhaust back-pressure. Therefore a more sophisticated CAC- 


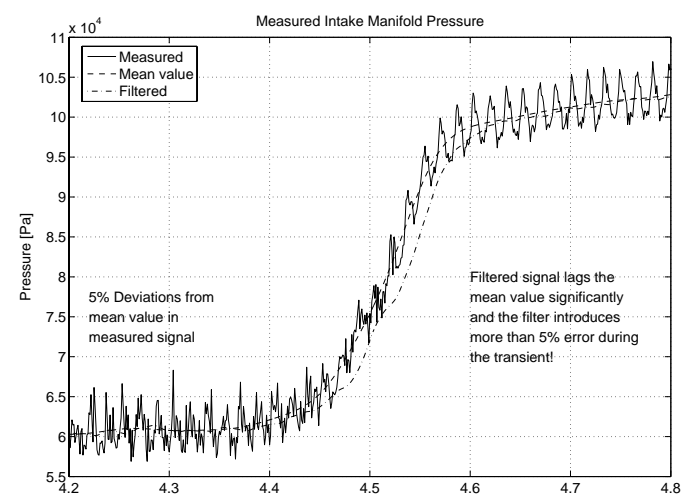

Fig. 2. Rapid tip-in at constant speed 1800 RPM. Measured intake manifold pressure is subjected to noise caused by engine pumpings and the electronics. Dampening the noise using a filter results in a significant lag compared to the meanvalue.

model, and thus controller, is beneficial (Andersson and Eriksson, 2004a).

For TC-engines a traditional control structure is suggested, with one feedforward controller and one slower feedback controller. The feedback controller maintains $\lambda=1$ at stationary conditions using a PIcontroller with feedback from a wide-band oxygensensor.

Here the focus is on design of the feedforward controller which estimates CAC at intake valve closing. It consists of two parts: An observer and a predictor. The observer is based on a mean value engine model and it estimates the system state, including the non measured exhaust manifold pressure, using feedback from measured intake manifold pressure and pressure after the intercooler. Then the estimated system state together with the mean value engine model are used to predict the CAC one revolution ahead. Using the suggested feedforward controller the error in air-fuel ratio during load transients are reduced from $30 \%$ down to only $5 \%$.

\section{MEAN VALUE ENGINE MODEL}

A component based mean value engine model suitable for air-fuel control forms the base of the feedforward design. Each component relies on physically based models of the underlying engine component.

In a TC engine both intake and exhaust systems are tightly coupled through the turbo, and Andersson and Eriksson (2004a) shows that CAC estimates are improved when the exhaust manifold pressure is included. In the CAC model, Equation (1), the coupling between the intake- and exhaust side is shown.

$$
\mathrm{CAC}=\frac{p_{\mathrm{im}} C_{\eta_{\mathrm{vol}}} V_{\mathrm{d}}\left(r_{c}-\left(\frac{p_{\mathrm{em}}}{p_{\mathrm{im}}}\right)^{\frac{1}{\gamma_{e}}}\right)}{\left(1+\frac{1}{\lambda\left(\frac{A}{F}\right)_{s}}\right)\left(r_{c}-1\right) R_{\mathrm{im}}\left(T_{\mathrm{im}}-C_{1}\left(\frac{1}{\lambda}-1\right)\right)}
$$

The nomenclature is that pressures are denoted $p$, temperatures $T$, air-mass flows $W$, and the subscripts show the location. Possible locations are: air-filter (af), compressor (comp), intercooler (ic), intake manifold (im), exhaust manifold (em), and after turbine (t). The turbocharger speed is denoted by $\omega_{\mathrm{TC}}$.

The modeling methodology of restrictions in series with adiabatic control volumes is applied, see (Eriksson et al., 2002) for a description of the methodology and (Andersson, 2005) for specific adaptions. To describe the necessary pressures and temperatures the following components along the air path are required: air filter, compressor, intercooler, throttle, engine, turbine, wastegate, turbocharger speed, and exhaust system. Between these components a control volume with pressure and temperature states are placed as shown in Figure 3.

In the model there are thirteen states and the state derivatives are described by nonlinear functions of states and inputs, see Equation (2). Inputs to the model are: Throttle plate angle $\alpha$, engine speed $N$, wastegate opening $\alpha_{\mathrm{wg}}$, normalized air-fuel ratio $\lambda$, and ambient conditions (pressure $p_{\mathrm{a}}$ and temperature $T_{\mathrm{a}}$ ). Outputs are states and signals that can be derived from the estimated states such as air-mass flows.

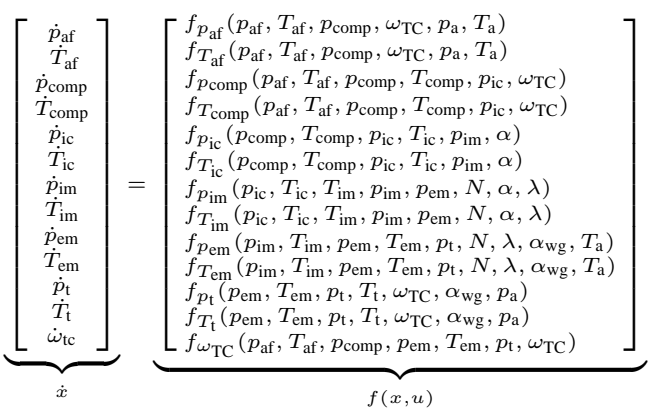

\section{AIR-FUEL CONTROLLER DESIGN}

The control objective is to maintain the normalized air-fuel ratio $\lambda$ close to one. During transients the challenge is the fast dynamics in the intake manifold requiring prediction of the CAC as shown in Figure 1. Here the air-fuel ratio control problem is partitioned into two parts, one feedforward controller to deal with the fast transients and one feedback controller to cancel stationary errors, see Figure 3. This division is standard in air-fuel ratio control (Guzzella, 1995; Powell et al., 1998; Chevalier et al., 2000).

\subsection{Observer Based Feedforward Control}

On NA-engines CAC is effectively described by an intake manifold pressure and temperature observer, but for turbocharged engines the $\mathrm{CAC}$ is more complex as it also involves non-measured signals on the exhaust side, as shown in Equation (1). Here this is handled 


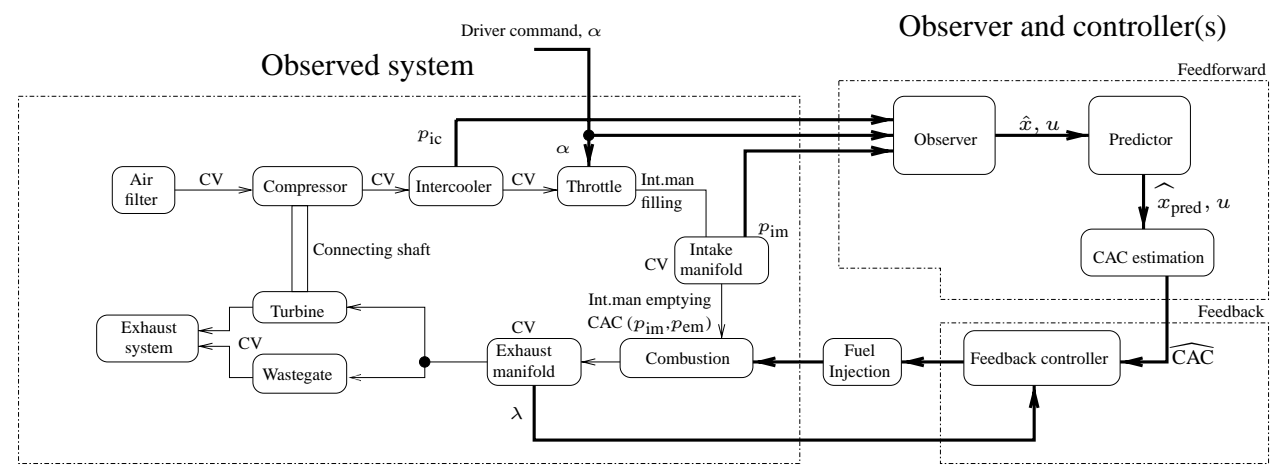

Fig. 3. An overview of a turbocharged SI-engine where the couplings between the intake and exhaust side are explicitly shown through the connecting shaft and the cylinder air charge (CAC). The observer estimates an initial condition to the predictor which estimates the system state one revolution ahead. The predicted state is used to estimate CAC (feedforward). Measured air-fuel ratio $\lambda$ is used as feedback to remove stationary errors. In the figure the letters CV means control volume, which represents a volume that connects two components. The control volume have states for pressure and temperature.

in a general way by introducing an observer that estimates the system state $\hat{x}(t)$ at time $t$. A predictor is added that aims at estimating the $\mathrm{CAC}$ at intake valve closing which is used to determine the mass of fuel to inject. The predictor uses the estimated system state vector together with the inputs to provide sufficient information to estimate CAC.

State Observer Design An observer,

$$
\dot{\hat{x}}=f(\hat{x}, u)+K(y-g(\hat{x}, u))
$$

with the measured outputs are $y=g(x, u)$, is developed. The gains $\mathrm{K}$ are determined offline for stationary points over the entire engine operating region. Gain switching is then performed online to select a precomputed $K$. When the observer is designed, the first step is to determine which feedback signals to use and to verify that the system is observable from the selected signals. In Andersson et al. (2005) it is shown that the system is locally structurally observable using feedback from arbitrary state(s). The available signals on the engine are: Air-mass flow after the air filter together with pressures and temperatures along the air-path. Pressure sensors are preferred for their fast dynamics and the following two sensors are chosen as feedback sources: Pressure after the intercooler $p_{\text {ic }}$ and intake manifold pressure $p_{\mathrm{im}}$. This is one of the suggested combinations in Andersson et al. (2005).

The observer gains are generated offline using a systematic design method (Andersson and Eriksson, $2004 b$ ). Outputs of the observer are the system states at time $t$ and these are used as initial conditions to a predictor. In Figure 4 a schematic of the implemented state observer is shown.

Predictor Design As prediction is necessary, the first challenge is to select the prediction horizon. It is determined by the sum of the computation time, injection time, and the time of the intake stroke. As the prediction is performed on a different computer there is a

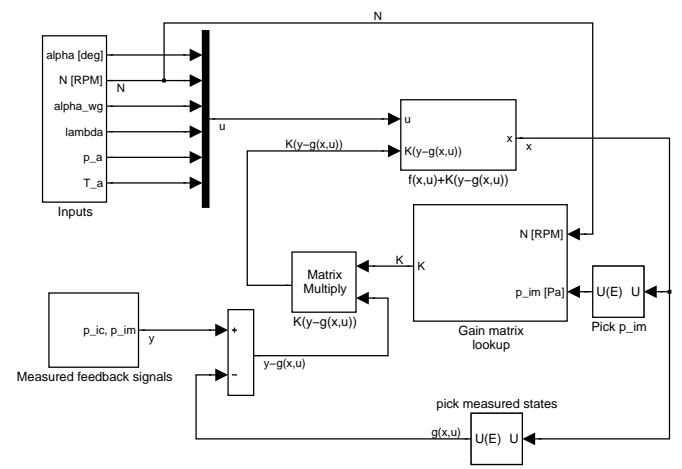

Fig. 4. A Simulink schematic of the implemented state observer.

transmission delay on the CAN-bus which has to be added. In the tests performed here the injection time is shorter than half a revolution, the intake stroke is half a revolution, and the computation time together with the delay on the CAN-bus are negligible. Thus the system state has to be predicted at time $t_{1}=t_{0}+\frac{60}{N}$, which is one revolution ahead. The state at $t_{1}$ is determined by simulating $\dot{\hat{x}}=f(\hat{x}, u)$ with the assumption that the input $u$ is constant between $t_{0}$ and $t_{1}$. The predictor uses the Runge-Kutta 4 method with a step size of $\frac{1}{400}$.

\section{$3.2 \lambda$-Feedback Control}

The purpose of the feedback controller is to compensate for stationary errors and it is implemented as a PI-style controller:

$$
e=\left(\lambda-\lambda_{\text {ref }}\right) \quad y=K\left(e+\int \frac{1}{T_{I}} e \mathrm{~d} t\right)
$$

In this case the output $y$ is limited and also the integrator is limited in order to avoid wind-up. The selection of $K$ and $\frac{1}{T_{I}}$ are not crucial and the following values were chosen: $K=0.25$ and $\frac{1}{T_{I}}=6$. 


\subsection{Total Controller}

The combined controller, with $\lambda_{\text {ref }}=1$, has the following appearance.

$$
m_{f}=\underbrace{\frac{\widehat{\mathrm{CAC}}}{\left(\frac{A}{F}\right)_{s}}}_{\text {Feedforward }} \times \underbrace{K\left((\lambda-1)+\int \frac{1}{T_{I}}(\lambda-1) \mathrm{d} t\right)}_{\text {Feedback }}
$$

\section{EXPERIMENTAL RESULTS}

The suggested controller has been tested in the research laboratory of Vehicular Systems on a 2 liter SAAB turbocharged SI-engine and the experimental setup is shown in Figure 5. The engine is controlled by an engine control unit (ECU) that communicates with a personal computer (PC) over a CAN-bus.

The observer and predictor is run in the PC and Equation (3) is implemented in MathWorks RealTime Workshop using Linux and RTAI 3.1 (Quaranta and Mantegazza, 2001; Rosenquist, 2003). In the PC it is also possible to measure analog signals. The PC estimates CAC, predicts it one revolution ahead, and then sends it to the ECU over the CAN-bus. Further it is possible to enable and disable prediction and feedback in the PC.

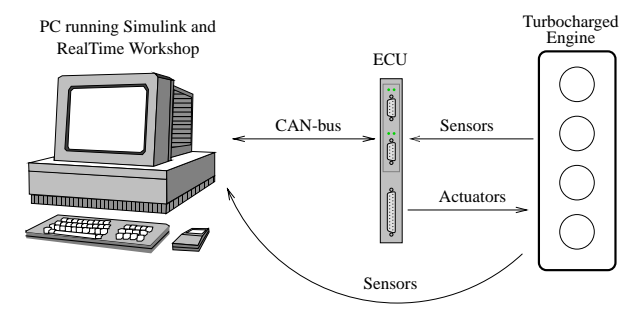

Fig. 5. The PC is running a realtime operating system (Linux with the RTAI patch) and communicates with the ECU over a CAN-bus. The ECU is responsible for sending sensor readings to the $\mathrm{PC}$ and also to inject the determined amount of fuel.

\subsection{Engine Experiments}

The performed experiment is a typical step in engine load at a constant speed of 2000 RPM. The same step has been repeated for the following four test cases:

(1) Purpose: Show the necessity of observer and predictor. Method: Use a conventional speeddensity feedforward controller with $\lambda$-feedback.

(2) Purpose: Show the necessity of the predicting feedforward step. Method: The controller is run with the predictor disabled.

(3) Purpose: Verify that prediction improves air-fuel ratio. Method: Run the controller with prediction and feedback enabled.
(4) Purpose: Estimate model errors. Method: Disable feedback but enable prediction.

In all experiments the engine has first been warmed up to operating temperature.

Conventional Speed-density Here the CAC is estimated as

$$
\mathrm{CAC}=\underbrace{\left(a_{0} p_{\mathrm{im}}+a_{1}\right)}_{\eta_{\mathrm{vol}} p_{\mathrm{im}}} \frac{V_{d}}{R_{\mathrm{im}} T_{\mathrm{im}}}
$$

which is suggested in Hendricks et al. (1996). To reduce noise in the measured intake manifold pressure signal, it is low-pass filtered using a fourth order butterworth filter with a cut-off frequency of $25 \mathrm{~Hz}$. The $\lambda$-feedback controller from Section 3.2 is used to maintain $\lambda=1$ for stationary conditions. This controller structure results in large $\lambda$-excursions of more than $30 \%$, which can be seen as a dashed line in Figure 6.

Observer Without Prediction The load step without prediction is shown as a dash-dotted line in Figure 6 and it is clear that there is a $10 \%$ error during the transient. The error is a result of that the fuel is injected based on a CAC estimate at $\mathrm{t}=\mathrm{IVO}$ minus the injection time. During transients the CAC changes substantially between this time and intake valve closing which cause $\lambda$-excursions, see for example Figure 1.

Observer With Prediction When the prediction step is enabled the transient error in $\lambda$ almost disappears as the solid line in Figure 6 is close to $\lambda=1$. This is a remarkable improvement especially as no fuel dynamics is included. However there are small errors left and possible sources are investigated in Section 4.3.

\subsection{Validation}

To validate the controller for a larger operating region, it was run at two different engine speeds. First it was run at 1500 RPM and then at 3000 RPM. In both cases the prediction time was one revolution. The results are shown in Figure 7. As the maximum error is still limited to $5 \%$ the power of the observer based feedforward controller is shown. 

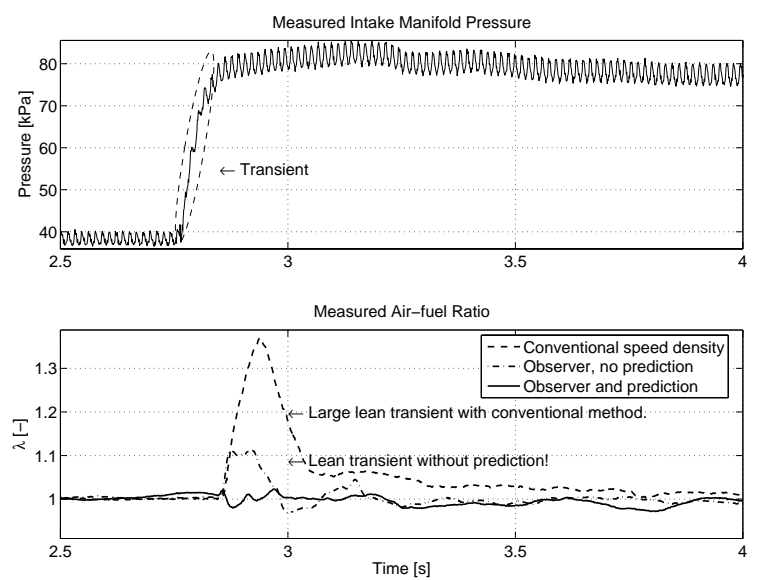

Fig. 6. Top: Intake manifold pressure. Bottom: Conventional speed density control results in a $37 \%$ error. Using the observer without prediction reduces the error to a $10 \%$ lean transient in $\lambda$ shown as a dash-dotted line. When the suggested observer is used together with prediction of the $\mathrm{CAC}$ one revolution ahead, the error decreases to approximately $3 \%$.
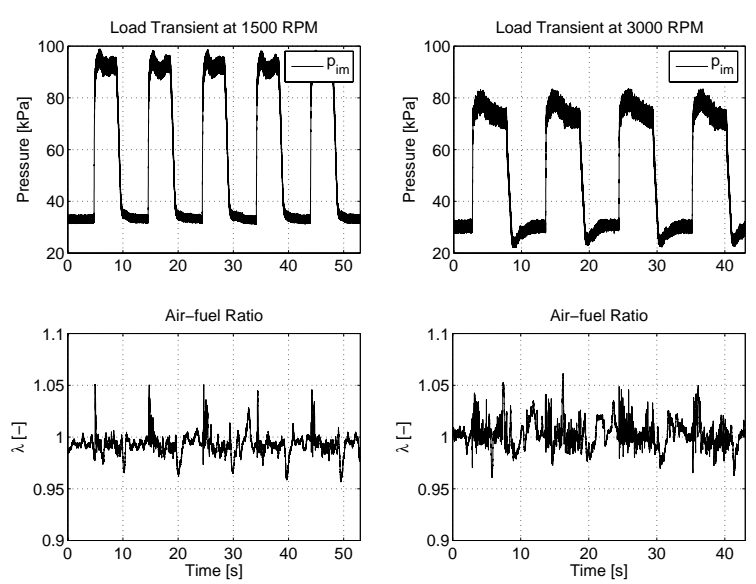

Fig. 7. Rapid load transients at 1500 RPM and 3000 RPM with the suggested observer based controller. Top: Intake manifold pressure. Bottom: Measured air-fuel ratio. During transients the resulting errors in $\lambda$ are less than $5 \%$ for both operating points. In the measurement at 3000 RPM the $\lambda$-excursion at $16 \mathrm{~s}$ in the lower right plot is caused by a disturbance on the throttle area signal.

\subsection{Sources of the Remaining Errors}

There are primarily three sources to the air-fuel ratio error:

- Prediction is made with the assumption of constant inputs which they are not.

- Model errors.

- No fuel dynamics included. However this effect is mostly pronounced at cold engine operation which is not considered here (Fozo and Aquino, 1988).

The two first items are discussed next.
Unknown Inputs There are two inputs that change during transients: Engine speed and throttle position. Normally the engine speed changes slowly which makes the assumption of constant speed during the prediction time valid. In this experiment the engine speed changes less than $3 \%$. The throttle on the other hand moves rapidly, which can be seen in Figure 8, and this contributes to a portion of the measured error in air-fuel ratio.
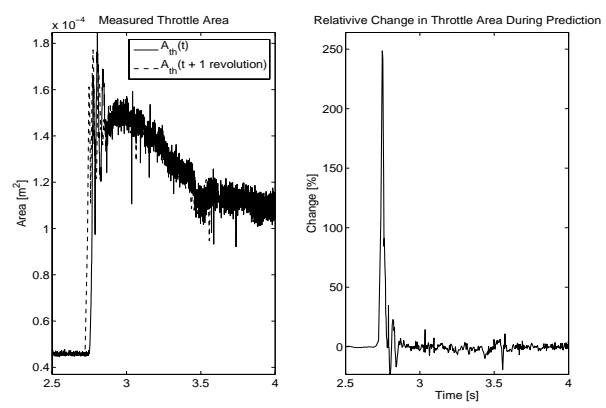

Fig. 8. The area can change significantly during the prediction time. During the transient the throttle movement can more than double the area!

Model Errors As CAC is only measurable for stationary conditions a test of the feedforward component, observer with prediction, was made by disabling the $\lambda$-feedback controller. The result is shown in Figure 9 . The stationary error differs before and after the step, both are less than 3\%. During the transient the measured error in air-fuel ratio is less than 5\% (worst case), which explains the accuracy of the model when used as a predictor. The errors are caused by model and parameter uncertainties.
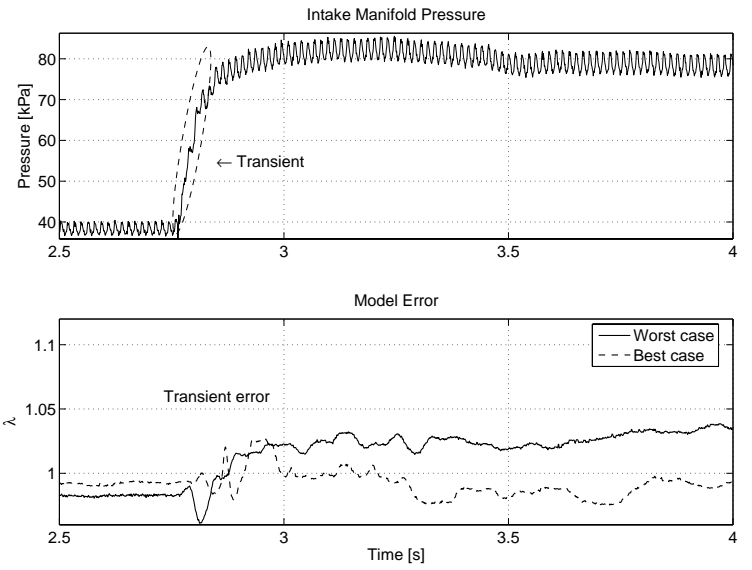

Fig. 9. Measured data with prediction enabled but with the feedback controller disabled. Several transients were run and in the best case the model describes CAC within $3 \%$ and in the worst case within $5 \%$. 


\section{CONCLUSIONS}

TC SI engines have a complex air-system which makes air-fuel ratio control a difficult task. An observer based control structure is proposed to reduce the deviations from $\lambda=1$ during transients. Two controllers are used, one feedforward and one feedback. The feedback controller cancels stationary errors using feedback from measured $\lambda$. The feedforward controller consists of an observer and a predictor which are based on a mean value engine model. The observer estimates an initial condition to the predictor which gives the system state one revolution ahead. The observer uses feedback from measured intake manifold pressure and measured pressure before the throttle. A systematic method is used to determine the observer feedback gains offline. In engine experiments the deviation from stoichiometric is reduced from more than $30 \%$ down to less than $5 \%$ over a wide range of engine speeds. Most of the improvement originates from the introduction of a model based observer.

\section{ACKNOWLEDGMENTS}

This work was financially supported by the Swedish Agency for Innovation Systems through the competence center ISIS. Also a special thanks to our research engineer Martin Gunnarsson who helped with the measurements.

\section{REFERENCES}

Andersson, Per (2005). A Component Based Mean Value Engine Model of a Turbocharged SIEngine. Technical report. Vehicular Systems, Department of Electrical Engineering. Linköpings Universitet.

Andersson, Per and Lars Eriksson (2004a). Cylinder Air Charge Estimator in Turbocharged SIEngines. Society of Automotive Engineers. SAE Technical Paper No. 2004-01-1366.

Andersson, Per and Lars Eriksson (2004b). Meanvalue observer for a turbocharged SI-engine. IFAC Symposium on Advances in Automotive Control. Salerno, Italy. pp. 146-151.

Andersson, Per, Erik Frisk and Lars Eriksson (2005). Structural Observability in Turbocharged Spark Ignited Engines - a Tool For Feedback Selection. Technical report. Vehicular Systems, ISY.

Bauer, Horst, Cypra, Arne, Beer, Anton and Bauer, Hans, Eds.) (1996). Bosch Automotive Handbook. 4 ed.. Robert Bosch Gmbh.

Chevalier, Alain, Christian Winge Vigild and Elbert Hendricks (2000). Predicting the port air mass flow of SI engines in air/fuel ratio control applications. In: Electronic Engine Controls. SP-1500. SAE Technical Paper No. 2000-01-0260.
Choi, Seibum B. and J. Karl Hedrick (1998). An Observer-Based Controller Design Method for Improving Air/Fuel Characteristics of Spark Ignition Engines. IEEE Transactions on Control Systems Technology 8(3), 325-334.

Eriksson, Lars, Lars Nielsen, Jan Brugård, Johan Bergström, Fredrik Pettersson and Per Andersson (2002). Modeling of a Turbocharged SI Engine. Annual Reviews in Control 26(1), 129-137.

Fozo, S. Robert and Charles F. Aquino (1988). Transient a/f characteristics for cold operation of a 1.6 liter engine with sequential fuel injection. SAE Technical Paper No. 880691.

Guzzella, Lino (1995). Models and Modelbased Control of IC-Engines - A Nonlinear Approach. SAE Technical Paper No. 950844.

Hendricks, Elbert, Alain Chevalier, Michael Jensen and Spencer C. Sorenson (1996). Modelling of the Intake Manifold Filling Dynamics. SAE Technical Paper No. 960037.

Heywood, John B. (1988). Internal Combustion Engine Fundamentals. McGraw-Hill International Editions.

Kiencke, Uwe and Lars Nielsen (2000). Automotive Control Systems. For Engine, Driveline, and Vehicle. Springer-Verlag.

Mondt, J.R. (2000). Cleaner Cars. The History and Technoloby of Emission Control Since the 1960s. SAE International.

Powell, J. David, N.P Fekete and Chen-Fang Chang (1998). Observer-based air-fuel control. Control Systems Magazine vol. 18(no. 5), pages 7283.

Quaranta, G. and P. Mantegazza (2001). Using MATLAB-Simulink RTW to Build Real Time Control Applications in User Space with RTAILXRT. Realtime Linux Workshop. Milano.

Rosenquist, Christer (2003). Hard realtime rapid prototyping development platform. Master's thesis. Linköpings Universitet, SE-581 83 Linköping. LiTH-ISY-EX-3377-2003.

Appendix A. NOMENCTLATURE

$\begin{array}{cl}\gamma_{e} & \begin{array}{l}\text { Ratio of specific heats on the exhaust } \\ \text { side }\end{array} \\ C_{\eta_{\mathrm{vol}}} & \begin{array}{l}\text { Parameter describing the pumping ca- } \\ \text { pabilities of the engine }\end{array} \\ \left(\frac{A}{F}\right)_{s} & \begin{array}{l}\text { Stoichiometric ratio of air and fuel } \\ V_{\mathrm{d}}\end{array} \\ r_{c} & \begin{array}{l}\text { Displacement volume of the engine } \\ \text { Compression ratio }\end{array} \\ C_{1} & \begin{array}{l}\text { Parameter describing additional } \\ \text { charge cooling for non-stoichiometric } \\ \text { conditions }\end{array}\end{array}$

